\title{
Using food science and immunology to hatch a better understanding of egg allergies.
}

\author{
Heidi Rowles* \\ Department of Allied Health, University of Cincinnati, Cincinnati, Ohio
}

\begin{abstract}
Egg allergies are a common food allergy and like all food allergies, are increasing in prevalence in the general population. Egg proteins are modified significantly from the raw or native form to the cooked form. These changes in conformation and binding sites present unique challenges when diagnosing and treating egg allergies. IgE and IgG testing of blood serum as well as skin prick tests in conjunction with oral food challenges can be used to perform comprehensive diagnostic egg allergy testing on an individual. One egg protein, ovomucoid, presents a specific complication in that it does not denature easily upon exposure to heat and digestion. An allergy to ovomucoid needs to be treated differently than an allergy to other egg proteins which denature more easily when exposed to heat and digestion. An individual who is allergic to ovomucoid is less likely to be able to consume cooked or raw egg or to outgrow their egg allergy. Identifying the specific protein(s) which elicit an allergic response is crucial when diagnosing and treating egg allergies.
\end{abstract}

Keywords: Food allergy, Food sensitivity, Egg allergy, Egg protein, Ovomucoid

Accepted on January 21, 2018

\section{Introduction}

Egg allergy is a common food allergy, especially in children [1]. As the number of people with food allergies increase, so does the number of food allergies caused by egg proteins [2]. While egg allergies are estimated to be present in $1-2 \%$ of the total population [3], they are more prevalent in children. In Australia, $8.9 \%$ of one-year-old children are affected by egg allergy [4]. Of adults with food allergies, $8 \%$ are allergic to eggs but an astounding $31 \%$ of children with food allergies are allergic to eggs [2]. The rate of anaphylaxis due to egg protein exposure is very low; it accounts for less than $10 \%$ of all foodrelated anaphylaxis [5]. Eggs are high in protein and there are differences in the number and structure of proteins in the egg white and the egg yolk. The egg white contains many more proteins than the yolk and is therefore responsible for a higher percentage of egg allergies and more commonly used to test for egg allergies [6-8].

\section{The immunology}

Proteins are responsible for allergic reactions. This is due to the allergenic protein binding with immunoglobulin's present in the human body [9]. The immune system is very complex and there are many different components involved, but only a basic understanding of $\operatorname{IgE}$ and $\operatorname{IgG}$ immunoglobulins and the difference between food allergy and food sensitivity are addressed in this article. At this time, allergies are typically tested for by measuring the blood serum levels of $\operatorname{IgE}$ and $\operatorname{IgG}$ immunoglobulin's [10]. There are other immunoglobulins such as $\operatorname{IgA}$ and $\operatorname{IgM}$ present in the body but their relevance in allergies is not yet understood [11]. IgE testing of a person's blood serum can detect an allergy to a specific protein $[12,13]$. Presence of $\operatorname{IgE}$ indicates that there is a true allergy and a severe reaction to exposure of the protein is likely $[12,14]$. An IgG test will detect the level of IgG immunoglobulin present in a blood sample. IgG is a memory protein and, when present, is indicative of exposure to the specific protein being tested for [15]. Presence of IgG indicates that there has been exposure to the specific protein and there is a possibility of an allergy or sensitivity, but it is also possible that no reaction may occur [10]. A Skin Prick Test (SPT) detects an immediate reaction to a protein by placing an allergen protein sample onto a person's skin and then scratching or pricking the protein into the skin [8]. While an SPT can be uncomfortable for the patient, it is a true determination of a reaction to a specific protein and the results typically correlate very closely with $\operatorname{IgE}$ blood test $[6,16]$. While an IgE test will show if a person possesses an $\mathrm{IgE}$ for a specific protein, it does not indicate whether or not a reaction will occur. Food allergies can be life-threatening and knowing whether or not and how strongly a person will react upon exposure to an allergen is important information when developing a treatment plan.

\section{The food science}

Despite the overall number of proteins in the egg white, there are four major proteins responsible for egg allergies. Ovalbumin accounts for $54 \%$ of total egg white protein, ovomucoid 11\%, ovotransferrin (conalbumin) $12 \%$ and lysozyme $3.5 \%[1,6]$. It is possible to be allergic to any or all of these proteins. Differentiation of the protein source of an egg allergy is important because egg proteins react differently to heat and digestion [7]. Ovalbumin, ovotransferrin, and lysozyme are easily denatured by heat and digestion [17]. Cooked forms of these proteins may be tolerated by someone who tests positive for an allergy to these proteins as the protein structure will be different in the cooked versus the native (raw) protein [18]. Ovomucoid, however, is heat and 
digestive resistant and it is less likely that a person with an allergy to this protein will be able to tolerate it in either the cooked or native form $[7,17,18]$. Tolerance of egg protein is indicative of whether or not a person will outgrow an egg allergy [16]. Persons who are allergic to ovomucoid are less likely to outgrow their allergy than persons who are allergic to ovalbumin, ovotransferrin, or lysozyme. For a person to avoid foods that contain the specific egg proteins to which they are allergic, it is important that they know specifically to which egg protein(s) they have an allergy.

\section{Discussion}

Ovomucoid contains nine disulphide bridges and a high content of helical and beta-pleated sheets which give it a very stable structure that is resistant to denaturation, even when cooked $[6,19]$. Of the egg white proteins, this stability is only known to be characteristic of the ovomucoid protein; the other proteins are more easily denatured when heated or exposed to digestion. This variation in protein stability provides a possibility for persons who are allergic to egg proteins other than ovomucoid to be able to consume cooked eggs [7]. Studies have shown that when a person who has an egg allergy is able to consume cooked eggs, that person has a higher probability of outgrowing their egg allergy altogether [3]. Evenpoel et al. showed that approximately $90 \%$ of cooked egg protein is digestible [20]. This percentage correlates with the percentage of ovomucoid in egg white, $11 \%$, not being digestible. This same study showed that only about $50 \%$ of raw egg is digestible [20]. Understanding how egg protein structures differ from native to cooked form can help understand this digestibility difference. Raw egg protein is globular [21]. As the egg protein is heated, it denatures or unfolds. As the egg is continued to be heated, the proteins aggregate, forming a structure that is altered from the native egg protein; it is more linear [22]. The binding sites of the cooked egg protein can be altered as well, affecting the protein's ability to bind to the IgE and IgG immunoglobulin's, which will affect the immunologic reaction. Understanding the difference in protein structure can help explain why cooked egg and raw egg have different levels of digestibility as well as varying allergic reactions. Raw egg contains the native egg proteins which elicit a higher percentage of allergic reactions than cooked egg [7]. Palmer et al. suggest that introducing cooked egg before raw egg could reduce allergenic reaction [4]. Some of the subjects in this study reacted to cooked egg but not to raw egg. A letter responding to this article suggested that processing of the egg may have different effects on food allergens, offering a possible explanation as to why these test subjects reacted to cooked egg but not raw egg [23]. Another plausible explanation could be that the root of the differences in outcomes is that the specific protein allergens were not known nor accounted for in this study; the measurements of allergy were only given as IgE or IgG-mediated reactions [4]. The test subjects in the Palmer study could have been allergic to ovomucoid, which would have elicited a reaction when these subjects were exposed to the cooked egg. The raw egg in this study was introduced in powder form and it is possible these two test subjects ingested less or no ovomucoid content in the powder than in the cooked egg they were given, lowering the probability of an allergic reaction. A confounding variable in the Palmer study was that the infants were given either rice powder or raw egg powder, and rice can be a food allergen. When a person has one food allergy, they are more likely to exhibit an allergic response to another food allergen [6]. Using rice powder as a control in this study could have caused test subjects who were allergic to rice to develop or exhibit allergic symptoms when exposed to the egg, skewing the true results of testing for egg allergy. The Palmer study did not account for breastfed babies who may have been exposed to egg protein through the breast milk. The study was designed to test whether early exposure to egg would help reduce egg allergy and the results showed that early exposure to egg did reduce the prevalence of egg allergy [4]. This study could have been more significant if confounding variables were eliminated by using a control powder that does not contain a food allergen and restricting the diets of breastfeeding mothers to ensure they did not consume any egg protein. Acid-reducing medications such as proton pump inhibitors are being prescribed for infants and children at an increasing rate [24]. Food allergen proteins will normally be broken down during digestion [15]. However, when proper digestion does not take place due to decreased acid in the stomach, these allergenic proteins and the immunoglobulin's they bind to have a higher probability of entering the bloodstream and inducing an immunologic reaction [15]. The immune system works in such a way that when a foreign entity such as a food molecule enters the body, the entity is investigated by immune cells and is either recognized by memory immune cells or identified as a new and unknown entity. When the entity is recognized, the immune system will either remember the molecule as harmless or as threatening, the latter launching an immune or allergic response [10,12,25]. When this process takes place in the digestive tract, the majority of the immunological proteins and food molecules will be digested with no adverse reactions or complications [26]. When this process takes place in a digestive tract that is not functioning properly, the risk of complication is elevated [25]. This could be a reason there is an increase in food allergies across the general population including adults. Millions of people take acid-reducing medications including proton pump inhibitors which can reduce digestion and therefore increase the risk of allergic reactions to food molecules [27].

\section{Conclusion}

A more comprehensive way to treat egg allergies would be to start with IgE test for high-risk persons. The IgE test will determine the specific protein(s) responsible for the allergy if there are any present. If the $\operatorname{IgE}$ test is negative but the individual shows a reaction to egg, an IgG test as well as a skin prick test (SPT) should be performed to determine if there is any immune-based response to the egg proteins. A person who tests positive to ovomucoid on an IgE screening should only be given an oral challenge in a medical setting equipped to handle anaphylaxis. A person who tests positive for other egg proteins such as ovalbumin, lysozyme, and ovotransferrin should be fed cooked egg first to determine if they are able to tolerate the cooked egg. If the person is able to tolerate cooked egg, after a period of time of cooked egg consumption that yields no allergic reaction, raw egg could be 
introduced in an oral challenge. If a person cannot consume raw egg without an allergic reaction, the cooked egg could continue to be consumed as long as the cooked egg yields no allergic response. As there is not an allergy test for cooked egg proteins, the only way to obtain immunological response data is through oral challenges and consumption.

As the number of people taking stomach acid-reducing medications continues to increase, this could be a reason for increasing prevalence of food allergies. Studies should be undertaken to investigate whether or not acid-reducing medicines cause food allergies. As there is no known cure for food allergies, causes for food allergies need to be thoroughly explored. Increase in food allergies leads to an increase in anaphylaxis and possible death. If reducing stomach acid via medications is found to be a cause in the rise of food allergies, alternative treatments such as probiotics and digestive enzymes for digestive tract symptoms need to be explored in an attempt to wane the incidence of food allergies.

\section{Conflict of Interest}

The author declares that there is no conflict of interest regarding the publication of this paper.

\section{References}

1. Kim J, Lee J, Han Y, et al. Special consideration is required for the component-resolved diagnosis of egg allergy in infants. Ann Allergy Asthma Immunol. 2014;112(1):53-57.

2. Fremont S, Kanny G, Nicolas JP, et al. Prevalence of lysozyme sensitization in an egg-allergic population. Allergy. 1997;52(2):224-228.

3. Clark A, Islam S, King Y, et al. A longitudinal study of resolution of allergy to well-cooked and uncooked egg. Clin Exp Allergy. 2011;41(5):706-712.

4. Palmer DJ, Metcalfe J, Makrides M, et al. Early regular egg exposure in infants with eczema: A randomized controlled trial. J Allergy Clin Immunol. 2013;132(2):387-392.

5. Tey D, Heine RG. Egg allergy in childhood: an update. Curr Opin Allergy Clin Immunol. 2009;9:244-250.

6. Caubet JC, Kondo Y, Urisu A, et al. Molecular diagnosis of egg allergy. Curr Opin Allergy Clin Immunol. 2011;11:210215 .

7. Rupa P, Schnarr L, Mine Y. Effect of heat denaturation of egg white proteins ovalbumin and ovomucoid on CD4 T cell cytokine production and human mast cell histamine production. J Functional Foods. 2015;18:28-34.

8. Mine Y, Yang M. Recent advances in the understanding of egg allergens: basic, industrial, and clinical perspectives. J Agric Food Chem. 2008;56(13):4874-4900.

9. Nirula A, Glaser SM, Kalled SL, et al. What is IgG4? A review of the biology of a unique immunoglobulin subtype. Curr Opin Rheumatol. 2011;23(1):119-124.

10. Schwarz A, Panetta V, Cappella A, et al. IgG and IgG4 to 91 allergenic molecules in early childhood by route of exposure and current and future IgE sensitization: Results from the Multicentre Allergy Study birth cohort. J Allergy Clin Immunol. 2016;138(5):1426-1433.

11. Yamaki K, Miyatake K, Nakashima T, et al. Intravenous IgA complexed with antigen reduces primary antibody response to the antigen and anaphylaxis upon antigen re-Exposure by inhibiting Th1 and Th2 activation in mice. Immunopharmacology and Immunotoxicology. 2014;36(5):316-328.

12. Lin J, Sampson H. The role of immunoglobulin E-Binding epitopes in the characterization of food allergy. Curr Opin Allergy Clin Immunol. 2009;9(4):357-363.

13. Platts-Mills TAE. The role of immunoglobulin $E$ in allergy and asthma. Am J Respir Crit Care Med. 2001;164(1).

14. Ahrens B, Schulz G, Borres MP et al. The role of hen's eggspecific IgE, IgG and IgG4 in the diagnostic procedure of hen's egg allergy. Allergy. 2010;65(12):1554-1557.

15. Gocki J, Bartuzi Z. Role of immunoglobulin G antibodies in diagnosis of food allergy. Postepy Dermatol Alergol. 2016;33(4):253-256.

16. Roches AD, Nguyen M, Paradis L, et al. Tolerance to cooked egg in an egg allergic population. Allergy. 2006;61:899900 .

17. Ortiz V, Pascal M, Jim R, et al. Ovalbumin-Specific IgE/ IgG4 ratio might improve the prediction of cooked and uncooked egg tolerance development in egg-Allergic children. Clin Exp Allergy. 2014;44: 579-588.

18. Senouf AHB, Borres MP, Eigenmann PA, et al. Native and denatured egg white protein IgE tests discriminate hens egg allergic from egg-tolerant children. Pediatr Allergy Immunol. 2015;26(1):12-17.

19. Roth K. Proteins present in egg white - part of the boiled eggs. ChemistryViews. 2012.

20. Evenepoel P Geypens B, Luypaerts A, et al. Digestibility of cooked and raw egg protein in humans as assessed by stable isotope techniques. J Nutr. 1998;128(10):1716-1722.

21. Chung WH, Lai KM, Hsu KC. Comparative study on histological structures of the vitelline membrane of hen and duck egg observed by cryo-scanning electron microscopy. J Agric Food Chem. 2010;58(3):1794-1799.

22. Alleoni A, Carraro C. Albumen protein and functional properties of gelation and foaming. Scientia Agricola. 2006;63(3):291-298.

23. Calvani M, Giorgio V, Sopo SM. Is cooked whole egg really less allergenic than pasteurized raw whole egg powder? J Allergy Clin Immunol. 2014;133(2):601.

24. Lun IC, Ali N, John T, et al. Proton pump inhibitor use in infants. J Pediatr Gastroenterol Nutr. 2012;54(1):8-14.

25. Astwood JD, Leach JN, Fuchs RL. Stability of food allergens to digestion in vitro. Nat Biotechnol. 1996;14(10):12691273. 
26. Fu T, Abbott UR, Hatzos C. Digestibility of food allergens and nonallergenic proteins in simulated gastric fluid and simulated intestinal fluid-a comparative study. J Agric Food Chem. 2002;50(24):7154-7160.

27. Brown T. The 10 most-prescribed and top-selling medications. WebMD. 2015.

\section{*Correspondence to:}

Heidi Rowles

Department of Allied Health

University of Cincinnati

Cincinnati

Ohio

Tel: 513-646-2599

E-mail: rowleshl@mail.uc.edu 